\title{
DISCRIMINACIÓN LGTBI EN LAS AULAS
}

\author{
Martínez-Gómez, N. \\ Dpto. de Psicología Básica, Clínica y Psicobiología \\ Universitat Jaume I, Castellón \\ gomezn@uji.es \\ Giménez-García, c. \\ Dpto. de Psicología Básica, Clínica y Psicobiología \\ Universitat Jaume I, Castellón \\ Enrique-Nebot, J. \\ Dpto. de Psicología Básica, Clínica y Psicobiología \\ Universitat Jaume I, Castellón \\ Elipe-Miravet, $\mathbf{M}$. \\ Dpto. de Psicología Básica, Clínica y Psicobiología \\ Universitat Jaume I, Castellón \\ Ballester-Arnal, $\mathbf{R}$. \\ 1 Dpto. de Psicología Básica, Clínica y Psicobiología \\ Universitat Jaume I,
}

Fecha de Recepción: 18 Febrero 2019

Fecha de Admisión: 30 Abril 2019

\section{RESUMEN}

Introducción: A pesar de que los grandes avances sociales, legales y culturales realizados en algunos países han facilitado una mayor aceptación de la diversidad sexual, el estigma asociado a la homosexualidad todavía juega un papel importante en el ajuste psicosocial de las personas con una orientación sexual alternativa a la heterosexual. Concretamente, estas personas se enfrentan todavía a múltiples barreras de la discriminación debido a su orientación sexual, tanto en el ámbito educativo como en el familiar. Por este motivo, el objetivo de este trabajo fue analizar las actitudes homofóbicas entre los adolescentes, así como el grado de victimización debido a la orientación sexual. Método: A tal fin, 87 estudiantes de secundaria (49.4\% chicas; $50.6 \%$ chicos) con edades entre los 13 y 16 años ( $M=13,34$; D.T.=,567) cumplimentaron un cuestionario ad-hoc sobre discriminación hacía el colectivo LGTBI. Resultados: En primer lugar, se observan diferencias significativas en función del género en cuanto al nivel de discriminación homofóbica $(t=2,47 ; p=, 015)$ : en concreto, los chicos muestran un mayor nivel de discriminación hacia personas LGTBI ( $M$ de57,56 en chicos frente a 49,34 en chicas [rango de 21 a 105]). En esta misma línea, ninguna de las chicas dijo haberse burlado de una persona LGTBI, frente al 13.2\% de los chicos que afirmó haberlo hecho 


\section{DISCRIMINACIÓN LGTBI EN LAS AULAS}

$\left(X^{2}=5,35 ; p=, 040\right)$. Analizando en concreto las distintas formas de discriminación homofóbica, afirmaciones donde los participantes obtuvieron una media mayor fueron "la mayoría de hombres gays que son insultados lo provocan por la forma en la que hablan, actúan o se visten" y "uno de los problemas con la discriminación es que algunas mujeres lesbianas no pueden aguantar una broma" (2,70 y 2,24 respectivamente [rango de 1 a 5]). En el extremo contrario, un porcentaje también significativamente mayor de hombres reportó haber sufrido amenazas (15,2\% hombres y $0 \%$ en mujeres $\left.\left[X^{2}=5,41 ; p=, 020\right]\right)$ o burlas $\left(10,5 \%\right.$ y $\left.0 \%\left[X^{2}=4,22 ; p=, 040\right]\right)$ debido a su orientación sexual. Conclusiones: Teniendo en cuenta los resultados, se propone elaborar estrategias para concienciar a los jóvenes heterosexuales acerca de su responsabilidad como aliados en la lucha por el respeto hacia personas LGTBI; así como en la prevención de la discriminación. Asimismo, se evidencia también la necesidad de elaborar estrategias para promover espacios seguros en centros educativos y abordar las situaciones derivadas del bullying homofóbico.

Palabras clave: diversidad afectivo-sexual; discriminación; bullying homofóbico; jóvenes

\section{ABSTRACT}

LGBTI discrimination in classrooms. Introduction: Although the great social, legal and cultural advances made in some countries have facilitated a greater acceptance of sexual diversity, the stigma associated with homosexuality still plays an important role in the psychosocial adjustment of people with a sexual orientation alternative to heterosexuality. In particular, these people still face multiple barriers of discrimination due to their sexual orientation, both in the educational and in the family context. For this reason, the aim of this work was to analyse homophobic attitudes among adolescents, as well as the degree of victimization due to sexual orientation. Method: To reach this objective, 87 high school students (49.4\% girls, $50.6 \%$ boys) aged between 13 and 16 years old ( $M$ $=13.34, S D=567$ ) completed an ad-hoc questionnaire on discrimination towards the LGTBI collective. Results: First, significant differences according to gender emerged on the homophobic discrimination level ( $t=2.47, p=, 015)$ : boys showed higher level of discrimination towards LGTBI people ( $M$ of 57.56 in boys versus 49.34 in girls [range of 21 to 105]). Similarly, none of the girls said to have played practical jokes to LGTBI people, compared to $13.2 \%\left(X^{2}=5.35, p=, 040\right)$. Analysing in particular the different forms of homophobic discrimination, statements where the participants obtained a higher average were "the majority of gay men who are insulted provoke it by the way they speak, act or dress" and "one of the problems with the discrimination is that some lesbian women can't tolerate a joke "(2.70 and 2.24 respectively [range of 1 to 5 ]). On the other hand, a significantly higher percentage of men reported having experienced threats $\left(15.2 \%\right.$ men and $0 \%$ in women $\left[X^{2}\right.$ $=5.41, p=, 020])$ or taunts $(10.5 \%$ and $\left.0 \%)\left[X^{2}=4.22, p=, 040\right]\right)$ due to their sexual orientation. Conclusions: Considering these results, we propose to develop strategies to raise awareness among heterosexual young people about their responsibility as allies in the struggle for respect for LGTBI people; as well as in the prevention of discrimination. Likewise, there is also evidence of the need to develop strategies to promote safe spaces in educational centres and to address situations derived from homophobic bullying.

Keywords: affective-sexual diversity; discrimination; homophobic bullying; young boys

\section{INTRODUCCIÓN}

A pesar de que los grandes avances sociales, legales y culturales realizados en algunos países han facilitado una mayor aceptación de la diversidad sexual, el estigma asociado a la homosexualidad todavía juega un papel importante en el ajuste psicosocial de las personas con una orientación sexual alternativa a la heterosexual (Morell, Capel y Gajardo, 2005). Concretamente, estas personas se enfrentan todavía a múltiples barreras de la discriminación debido a su orientación sexual, tanto 
en el ámbito educativo como en el familiar. Según la UNESCO (2012) el bullying homofóbico, entendido como el maltrato psicológico, verbal o físico producido en el entorno escolar por motivos LGTBI (Platero y Gómez, 2007), es un problema generalizado en la mayor parte de escuelas del mundo. Más concretamente el $47 \%$ de la población europea lesbiana, gay, transexual, bisexual e intersexual y el 38\% de la española se siente discriminada (Grupo Educación COGAM, 2013).

Entre los factores que favorecen este tipo de discriminación, se destacan principalmente dos, por un lado, la interiorización de los estereotipos relacionados con las personas LGTBI; por otro lado, el establecimiento de comportamientos sexistas en la sociedad. Para autores como Soriano y Lázaro (2007), el hecho de que existan prejuicios hacia las personas que no son heterosexuales y se les otorgue una serie de atributos y formas de interactuar con la sociedad, hace que algunas personas heterosexuales rechacen todos aquellos patrones que se salgan del género normativo. Además, no solo a aquellas personas que son LGTBI sino también a aquellas personas que sus patrones coincidan con los asociados este colectivo, sin necesidad de pertenecer al mismo. Este fenómeno recibe el nombre de plumofobia. Por otro lado, autores como Basow y Johnson (2000), defienden que el género es entendido como un dispositivo social, convirtiéndose en un mecanismo de jerarquización y privilegios donde la discriminación viene dada por el no cumplimiento de las expectativas según una persona nace hombre 0 mujer.

Otros autores afirman (Morell y Gajardo, 2005) que además de estos dos factores, también influyen otros individuales en el Bullying homofóbico, como el miedo a convivir con lo diferente, conflictos con la propia sexualidad, compensar una baja autoestima con ser superior a otras personas, focalizar la atención en otras personas para ocultar la propia sexualidad o la necesidad de pertenencia al grupo.

La adolescencia se trata del periodo fundamental para establecer la identidad de las personas, si en este proceso identitario, las personas sufren discriminación y rechazo se puedan dar lugar a consecuencias negativas a nivel psicosocial (DiFulvio, 2011). Entre ellas destacan baja autoestima, ansiedad, depresión y problemas relacionados con el establecimiento de lazos afectivos (Almeida, 2009; Marchueta, 2014; Pichardo, 2012). Estas secuelas psicológicas se suelen mantener en el tiempo como un recuerdo desagradable y traumático, pudiendo desencadenar en homofobia interiorizada y síntomas asociados al estrés post-traumático.

Uno de los principales escenarios de la adolescencia son los centros educativos (Catwright 2006). Por ese motivo, el objetivo de este trabajo es evaluar la experiencia de discriminación en estudiantes de centros educativos de secundaria, en concreto, analizando las actitudes homofóbicas entre adolescentes, así como el grado de victimización debido a la orientación sexual.

\section{MÉTODO}

\section{Participantes}

Un total de 87 estudiantes de $2^{0}$ de Educación Secundaria Obligatoria de Castellón (España) participaron en el presente estudio. Las edades del alumnado estaban comprendidas entre los 13 y los 16 años, con una edad promedio de 13,34 años (DT=0,56), siendo el $50,6 \%$ chicas y el $49,4 \%$ chicos. El 89,8\% refirieron sentir atracción sexual/emocional hacia el sexo contrario, el 3,4 hacia ambos, el 3,4\% hacia nadie, y el 3,4\% no lo tenía claro.

\section{Instrumentos}

Todos los participantes respondieron un cuestionario ad hoc sobre LGTBfobia y discriminación. Este instrumento consta de 52 ítems con distintos formatos de respuesta que exploran la experiencia del nivel de conocimientos, actitudes, conductas y creencias. 


\section{DISCRIMINACIÓN LGTBI EN LAS AULAS}

Para el presente artículo se seleccionaron dos escalas:

- Actitudes y creencias hacia el colectivo LGTB. Esta escala está formada por 28 ítems en el que se presentan una serie de afirmaciones sobre actitudes y creencias hacia dicho colectivo. Los participantes han de contestar en base a una respuesta tipo Likert, siendo 1=Totalmente en desacuerdo y $5=$ Totalmente de acuerdo. La puntuación total puede ir de 28 a 140 puntos, mostrándose mayor LGTBfobia cuando mayor es la puntuación. La presente escala muestra una buena consistencia interna $(=0,883)$.

- Vivencias de discriminación debido a la orientación o la identidad sexual. Esta escala consta de 4 ítems dicotómicos, en los que se pregunta si alguna vez han presenciado discriminación (ítem 1), han defendido a otra persona de algún tipo de discriminación (ítem 2), han realizado algún acto discriminatorio (ítem 3) o han sido víctimas de los mismos (ítem 4). La consistencia interna de la escala es de 0,57 según el Alpha de Cronbach.

\section{Procedimiento}

Los datos fueron recogidos a través de un formulario online que se administró en un aula de informática del centro, asegurando un espacio suficiente entre el alumnado, para garantizar su intimidad. Una vez accedían al formulario online, se les informaba del carácter anónimo, voluntario y confidencial de la investigación, y se les pedía el consentimiento informado.

\section{Análisis estadísticos}

Para realizar los diferentes análisis se utilizó el programa estadístico SPSS versión 25. En primer lugar, se analizaron frecuencias y descriptivos para obtener los datos sociodemográficos. Asimismo, para analizar las diferencias entre chicos y chicas en la puntuación total en la escala de LGTBfobia, así como con los ítems de la escala, se realizaron pruebas t de Student. Por otra parte, para calcular las diferencias entre chicos y chicas en las experiencias vividas con la discriminación debido a la orientación o identidad sexual, se realizaron tablas de contingencias y pruebas de Chi cuadrado.

\section{RESULTADOS}

En términos generales, en los ítems sobre LGTBfobia que mayor acuerdo se ha observado han sido "Antes las personas estaban discriminadas por su orientación o identidad sexual, pero ahora ya no", "La mayoría de hombres gays que son insultados lo provocan por la forma en la que hablan, actúan o se visten", "Los travestis son hombres gays disfrazados de mujer", "Aceptaría la bisexualidad de mi pareja" y "Luchar contra la homofobia en los centros educativos es una labor del profesorado y el alumnado homosexual, que son los que se encuentran implicados" (véase tabla 1).

En cuanto a las diferencias entre chicos y chicas en los ítems sobre LGTBfobia, se observaron diferencias significativas, a nivel estadístico, en "La sola idea de sentirme atraído/a físicamente por personas de mi propio sexo me incomoda", "Respeto a los hombres gays, siempre y cuando no se exhiban", "Me siento a gusto si me ven en público con una persona claramente homosexual (inverso)", "Luchar contra la homofobia en los centros educativos es una labor del profesorado y el alumnado homosexual, que son los que se encuentran implicados", "No iría a lugares de ambiente, en los que se reúnen personas homosexuales, bisexuales y transexuales, por miedo a que me acosen", "Aceptaría la bisexualidad de un hijo (inverso)", "Sería chocante ver a una persona trans besándose con otra persona" y "Uno de los problemas con la discriminación es que algunas mujeres lesbianas no pueden aguantar una broma". En todos los ítems muestran mayor acuerdo los chicos, a excepción del ítem "Luchar contra la homofobia en los centros educativos es una labor del profesorado y el alumnado homosexual, que son los que se encuentran implicados", donde mostraban mayor acuerdo las chicas. 
Tabla 1. Diferencias entre chicos y chicas en los ítems sobre LGTBfobia.

\begin{tabular}{|c|c|c|c|c|}
\hline & Total & Chicos & Chicas & \\
\hline & M (DT) & M (DT) & M (DT) & $\mathrm{t}(\mathrm{p})$ \\
\hline $\begin{array}{l}\text { Ítem } 1 . \text { La sola idea de sentirme atraído/a } \\
\text { físicamente por personas de mi propio sexo me } \\
\text { incomoda. }\end{array}$ & $2,25(1,34)$ & $2,59(1,45)$ & $1,91(1,15)$ & $2,43(0,017)$ \\
\hline $\begin{array}{l}\text { *Ítem } 2 \text {. Está bien que los hombres gays puedan } \\
\text { adoptar hijos. }\end{array}$ & $1,29(0,90)$ & $1,25(0,75)$ & $1,33(1,04)$ & $-0,38(0,698)$ \\
\hline $\begin{array}{l}\text { Ítem 3. Podría llegar a insultar a una persona de } \\
\text { mi mismo sexo si intentara ligar conmigo. }\end{array}$ & $1,87(1,16)$ & $2,09(1,19)$ & $1,65(1,11)$ & $1,77(0,079)$ \\
\hline $\begin{array}{l}\text { Ítem 4. Las personas trans no deberían enseñar } \\
\text { en las escuelas. }\end{array}$ & $1,11(0,41)$ & $1,14(0,40)$ & $1,09(0,42)$ & $0,48(0,629)$ \\
\hline $\begin{array}{l}\text { Ítem } 5 . \text { Las personas bisexuales son promiscuas } \\
\text { y viciosas. }\end{array}$ & $1,71(0,86)$ & $1,89(0,92)$ & $1,53(0,76)$ & $1,93(0,056)$ \\
\hline $\begin{array}{l}\text { Ítem 6. Las relaciones sexuales entre mujeres } \\
\text { lesbianas no son plenamente satisfactorias. }\end{array}$ & $1,91(0,94)$ & $1,91(1,01)$ & $1,91(0,89)$ & $0,01(0,992)$ \\
\hline $\begin{array}{l}\text { Ítem } 7 . \text { Respeto a los hombres gays, siempre y } \\
\text { cuando no se exhiban. }\end{array}$ & $2,13(1,42)$ & $2,61(1,55)$ & $1,63(1,09)$ & $3,42(0,001)$ \\
\hline $\begin{array}{l}\text { Ítem } 8 \text {. Sería chocante ver a dos mujeres } \\
\text { lesbianas besándose. }\end{array}$ & $1,41(0,97)$ & $1,50(1,02)$ & $1,33(0,91)$ & $0,83(0,405)$ \\
\hline $\begin{array}{l}\text { *Ítem } 9 . \text { Me siento a gusto si me ven en público } \\
\text { con una persona claramente homosexual. }\end{array}$ & $1,94(1,24)$ & $2,39(1,22)$ & $1,49(1,09)$ & $3,59(0,001)$ \\
\hline $\begin{array}{l}\text { İtem } 10 \text {. No defendería a una persona } \\
\text { homosexual de un insulto por si piensan que yo } \\
\text { también lo soy. }\end{array}$ & $1,34(0,77)$ & $1,50(0,92)$ & $1,19(0,54)$ & $1,92(0,058)$ \\
\hline $\begin{array}{l}\text { Ítem 11. Antes las personas estaban } \\
\text { discriminadas por su orientación o identidad } \\
\text { sexual, pero ahora ya no. }\end{array}$ & $2,85(1,22)$ & $2,64(1,14)$ & $3,07(1,28)$ & $-1,66(0,099)$ \\
\hline $\begin{array}{l}\text { Ítem 12. Luchar contra la homofobia en los } \\
\text { centros educativos es una labor del profesorado y } \\
\text { el alumnado homosexual, que son los que se } \\
\text { encuentran implicados. }\end{array}$ & $2,45(1,42)$ & $2,14(1,25)$ & $2,77(1,52)$ & $-2,11(0,038)$ \\
\hline $\begin{array}{l}\text { Ítem 13. No iría a lugares de ambiente, en los } \\
\text { que se reúnen personas homosexuales, } \\
\text { bisexuales y transexuales, por miedo a que me } \\
\text { acosen. }\end{array}$ & $1,82(1,14)$ & $2,32(1,32)$ & $1,30(0,59)$ & $4,62(0,000)$ \\
\hline $\begin{array}{l}\text { Ítem } 14 \text {. Sería chocante ver a dos hombres gays } \\
\text { besándose. }\end{array}$ & $1,51(0,97)$ & $1,68(1,07)$ & $1,33(0,83)$ & $1,72(0,088)$ \\
\hline *Ítem 15. Aceptaría la bisexualidad de un hijo. & $1,62(1,13)$ & $1,93(1,28)$ & $1,30(0,86)$ & $2,69(0,009)$ \\
\hline $\begin{array}{l}\text { Ítem 16. Las mujeres lesbianas que se muestran } \\
\text { más masculinas es porque en el fondo quieren }\end{array}$ & $2,14(1,17)$ & $2,07(1,10)$ & $2,21(1,24)$ & $-0,55(0,578)$ \\
\hline
\end{tabular}
ser hombres. 


\section{DISCRIMINACIÓN LGTBI EN LAS AULAS}

Ítem 17. Las relaciones sexuales con personas trans no son plenamente satisfactorias.

*Ítem 18. Aceptaría la bisexualidad de mi pareja.

Ítem 19. Si un hombre tiene sentimientos homosexuales debería hacer todo lo posible por superarlos.

*Ítem 20. Está bien que las personas trans puedan adoptar hijos.

Ítem 21. Las personas bisexuales son indefinidas e indecisas.

Ítem 22. Las relaciones sexuales entre hombres gays no son plenamente satisfactorias.

Ítem 23. Sería chocante ver a una persona trans besándose con otra persona.

Ítem 24. Uno de los problemas con la discriminación es que algunas mujeres lesbianas no pueden aguantar una broma.

Ítem 25. Los travestis son hombres gays disfrazados de mujer.

*Ítem 26. Está bien que las mujeres lesbianas puedan adoptar hijos.

Ítem 27. La mayoría de hombres gays que son insultados lo provocan por la forma en la que hablan, actúan o se visten.

Ítem 28. Para estar con una mujer lesbiana masculina, estaría con un hombre.

\begin{tabular}{|c|c|c|c|}
\hline $2,16(1,14)$ & $2,07(1,06)$ & $2,26(1,21)$ & $-0,76(0,446)$ \\
\hline $2,53(1,56)$ & $2,64(1,67)$ & $2,42(1,45)$ & $0,64(0,519)$ \\
\hline $1,99(1,45)$ & $2,27(1,53)$ & $1,70(1,31)$ & $1,87(0,064)$ \\
\hline $1,22(0,76)$ & $1,25(0,78)$ & $1,19(0,76)$ & $0,38(0,701)$ \\
\hline $1,89(0,95)$ & $1,98(1,00)$ & $1,79(0,91)$ & $0,90(0,367)$ \\
\hline $2,22(1,12)$ & $2,41(1,22)$ & $2,02(0,98)$ & $1,61(0,110)$ \\
\hline $1,60(1,03)$ & $1,86(1,13)$ & $1,33(0,86)$ & $2,49(0,015)$ \\
\hline $2,24(1,32)$ & $2,66(1,44)$ & $1,81(1,05)$ & $3,12(0,003)$ \\
\hline $2,56(1,43)$ & $2,77(1,44)$ & $2,35(1,41)$ & $1,38(0,170)$ \\
\hline $1,24(0,82)$ & $1,39(0,97)$ & $1,09(0,61)$ & $1,69(0,095)$ \\
\hline $2,70(1,60)$ & $2,91(1,58)$ & $2,49(1,62)$ & $1,22(0,224)$ \\
\hline $1,80(1,22)$ & $1,73(1,14)$ & $1,88(1,31)$ & $-0,59(0,555)$ \\
\hline
\end{tabular}

Por lo que respecta a la puntuación total de la escala de LGTBfobia, la media de la muestra total fue de 53,50 (DT=15,92). En función del sexo, se observa que los chicos puntúan más alto $(\mathrm{M}=57,56 ; \mathrm{DT}=15,95)$ que las chicas $(\mathrm{M}=49,34 ; \mathrm{DT}=14,96)$, siendo las diferencias estadísticamente significativas ( $t=2,47 ; p=0,015)$.

En cuanto a la experiencia vivida de discriminación, en niveles generales se observa que hay más gente ha observado discriminaciones LGTB frente a los que las han defendido, siendo lo más frecuente el rol de espectadores (ver tabla 2). Por otro lado, se observa que en todas las agresiones menos las físicas, hay personas que reportan haberlas realizado, pero destacamos el hecho de que en todos los tipos hay personas que las han sufrido.

Si observamos los tipos de agresión más frecuentes como víctima vemos que las más sufridas son comentarios negativos, seguidas de insultos, amenazas, burlas, ignorar y agresiones físicas. Mientras que como agresores nos encontramos en primer lugar con comentarios negativos, seguidas de ignorar, insultos, burlas amenazas y ninguna agresión física. Finalmente, como espectadores nos encontramos en primer lugar con las burlas seguido de amenazas, comentarios negativos, insultos, agresiones físicas y por último ignorar. 
Tabla 2. Puntuaciones en las diferentes formas de discriminación LGTBI.

\begin{tabular}{ccccc}
\hline & $\begin{array}{c}\text { TOTAL } \\
(\%)\end{array}$ & $\begin{array}{c}\text { Chicos } \\
(\%)\end{array}$ & $\begin{array}{c}\text { Chicas } \\
(\%)\end{array}$ & Chi2 (p) \\
\hline Agresiones físicas & & & & \\
He presenciado & 78,3 & 83,3 & 72,7 & $1,13(0,286)$ \\
He defendido & 37,7 & 36,1 & 39,4 & $0,07(0,779)$ \\
He realizado & 0 & 0 & 0 & \\
Me han realizado & 1,4 & 2,8 & 0 & $0,93(0,335)$ \\
Amenazas & & & & \\
He presenciado & 83,3 & 84,8 & 81,8 & $0,10(0,741)$ \\
He defendido & 42,4 & 42,4 & 42,4 & $0,00(1,000)$ \\
He realizado & 1,5 & 3 & 0 & $1,01(0,314)$ \\
Me han realizado & 7,6 & 15,2 & 0 & $5,41(0,020)$ \\
Insultos & & & & \\
He presenciado & 80 & 92,1 & 67,6 & $7,05(0,008)$ \\
He defendido & 44 & 34,2 & 54,1 & $2,99(0,083)$ \\
He realizado & 5,3 & 5,3 & 5,4 & $0,01(0,978)$ \\
Me han realizado & 10,7 & 15,8 & 5,4 & $2,12(0,145)$ \\
Burlas & & & & \\
He presenciado & 89,5 & 92,1 & 86,8 & $0,55(0,455)$ \\
He defendido & 36,8 & 31,6 & 42,1 & $0,90(0,342)$ \\
He realizado & 5,3 & 10,5 & 0 & $4,22(0,040)$ \\
Me han realizado & 6,6 & 13,2 & 0 & $5,35(0,021)$ \\
Comentarios negativos & & & & \\
He presenciado & 81,8 & 83,8 & 80 & $0,18(0,667)$ \\
He defendido & 49,4 & 40,5 & 57,5 & $2,21(0,137)$ \\
He realizado & 10,4 & 10,8 & 10 & $0,01(0,907)$ \\
Me han realizado & 13 & 13,5 & 12,5 & $0,01(0,895)$ \\
Ignorar & & & & \\
He presenciado & 73,6 & 82,9 & 64,9 & $2,99(0,083)$ \\
He defendido & 45,8 & 48,6 & 43,2 & $0,20(0,650)$ \\
He realizado & 6,9 & 5,7 & 8,1 & $0,15(0,690)$ \\
Me han realizado & 5,6 & 11,4 & 0 & $4,47(0,034)$ \\
\hline & & & &
\end{tabular}

Por último, en cuanto a la diferenciación por sexo, vemos que los chicos son los que mayor discriminación sufren por su orientación sexual, siendo las diferencias significativas con las chicas en las amenazas, en las burlas y en ser ignorado. También se observaron diferencias significativas en las burlas realizadas y en los insultos que se han presenciado, siendo más frecuentes en los chicos.

\section{CONCLUSIONES}

El hecho de que exista esta diferencia entre la discriminación realizada y la percibida, consideramos que se puede dar por dos supuestos, uno sería el hecho de que las personas se vean influenciadas por la deseabilidad social a la hora de contestar el cuestionario y por otro lado el hecho de que las personas que ejercen la discriminación no sean conscientes que la está realizando. Por otro lado, encontramos como espectadores en primer lugar con las burlas seguido de amenazas, comentarios negativos, insultos, agresiones físicas y por último ignorar. Lo que nos refuerza la idea de tener que fortalecer la figura de la persona heterosexual como aliada al colectivo LGTBI, así como hacerles ver la implicación y responsabilidad del rol del espectador en las situaciones de bullying (Trautmann, 2008).

Son muchas las personas que sufren discriminación, sobre todo en el ámbito educativo, lo que hace que el hecho de acudir a las aulas sea algo traumático y produzca secuelas a largo plazo (Marchueta, 2014). Es importante reducir estas situaciones en los centros educativos y para ello es 


\section{DISCRIMINACIÓN LGTBI EN LAS AULAS}

necesario que aquellas personas que ejercen este tipo de discriminación, sean conscientes de las consecuencias negativas de su comportamiento, así como dotar de herramientas a todo el estudiantado y profesorado para poder intervenir antes este tipo de situaciones (Cerezo, 2015).

Actualmente en el sistema educativo nos encontramos con un enfoque heterosexista en los materiales que se utilizan en las aulas, lo que hace que la falta de representación del colectivo en dichos materiales sea ofensiva y perjudique en el aprendizaje, puesto que las personas no heterosexuales en el aula representan una minoría significativa en la sociedad y este hecho es un reflejo de la discriminación e invisibilización de este colectivo. Como respuesta a ese hecho, nace la pedagogía Queer (Del Castillo, 2015), como propuesta de modelo pedagógico para prevenir y desmontar la heteronormatividad (todas las personas son heterosexuales hasta que se demuestre lo contrario) en las aulas, sobre todo quiere romper con el binomio normal-anormal, promoviendo la reflexión y la conciencia crítica. Esta heteronormatividad de la sociedad produce que la persona no heterosexual transite por diferentes fases de autoaceptación (Maroto, 2006) generando malestar y confusión en cuanto al desarrollo de su sexualidad. Este tipo de pedagogía aboga por cuestionar y tocar conciencia sobre los privilegios que se establecen en función de las identidades sexuales y de género. De forma que se trata de un intento por conseguir aulas libres y críticas, donde se valoren las diversidades. Obteniendo como resultado la eliminación de la dicotomía sexo-género, así como poder realizar análisis más amplios del género y de la diversidad en la adolescencia.

Por ello se propone elaborar estrategias para concienciar a los jóvenes heterosexuales acerca de su responsabilidad como aliados en la lucha por el respeto hacia personas LGTBI; así como en la prevención de la discriminación. Asimismo, se evidencia también la necesidad de elaborar estrategias para promover espacios seguros en centros educativos y abordar las situaciones derivadas del bullying homofóbico. Con la finalidad trabajar la realidad que vive el colectivo LGTBI de forma transversal en el currículum escolar y dotar así de conocimientos sobre diversidad afectivo sexual y de género. Para ello una de las finalidades es hacer ver y cuestionar los privilegios instaurados en la heteronormatividad. Todo ello fomentando la reflexión crítica y fortaleciendo los valores tolerantes, inclusivos y enriquecedores de la diversidad, con el fin de conseguir aulas libres y diversas.

\section{REFERENCIAS BIBLIOGRÁFICAS}

Almeida, J., Johnson, R., Corliss, H., Molnar, B. y Azrael, D. (2009). Emotional distress among LGBT youth: The influence of perceived discrimination based on sexual orientation. Journal of youth and adolescence, 38(7), 1001-1014.

Basow, S. A., y Johnson, K. (2000). Predictors of homophobia in female college students. Sex Roles, 42(5-6), 391-404.

Cartwright, I. (2006). Mind the gap: an investigation into perceived diversity gap in the English language learning sub-culture. Language Issues, 18(2), 176-183.

Cerezo, F. (2015). Bullying homofóbico. El papel del profesorado. International Journal of Developmental and Educational Psychology. Revista INFAD de Psicología., 1(1), 417-424.

Del Castillo, V. (2015). Heteronormativity and Classroom Strategies: An Educator's Guide to Queering the Normal. Recuperado de https://www.academia.edu/6035720/Heteronormativity and Classroom Strategies.

DiFulvio, G. T. (2011). Sexual minority youth, social connection and resilience: From personal struggle to collective identity. Social Science \& Medicine, 72(10), 1611-1617.

Grupo Educación COGAM (2013). Homofobia en las aulas, 2013. ¿Educamos en la diversidad afectivo sexual?. Recuperado de https://www.cop-cv.org/db/docu/160511115453TiAcC5TrGLQP.pdf

Marchueta, A. (2014). Consecuencias del bullying homofóbico retrospectivo y los factores psicoso- 
ciales en el bienestar psicológico de sujetos LGB. Revista de Investigación Educativa, 32(1), 255-271.

Maroto, A. (2006). Homosexualidad y Trabajo Social. Herramientas para la reflexion e intervencion profesional.

Morell, Capel Silvia y Daniel Gajardo (2005). "Consecuencias psicológicas del “Bullying” homofóbico y transfóbico: factores identificativos y preventivos". Artículo presentado en forma de taller en el IV Seminario de la AMPGIL (Associació de mares, pares de gays i lesbianes) celebrado el 12 de noviembre de 2005 en Barcelona, Espana.

Pichardo, J.I. (2013). Diversidad sexual y convivencia: una oportunidad educativa. Recuperado de www.felgtb.org/rs/3660/d112d6ad-54ec-438b-9358-4483f9e98868/234/filename/informe-finaldiversidad-y-convivencia.pdf

Platero, R. y Gómez, E. (2007). Herramientas para combatir el bullying homofóbico. Recuperado de: http://webs.ucm.es/BUCM/escritores/platero/obras/obr2856.pdf

Soriano, S. y Lázaro, S. (2007) Bullying homofóbico. Situación actual y características de la violencia escolar. Almería: Grupo Editorial Universitario. (169-174)

Trautmann M. (2008). Maltrato entre pares o "bullying": Una visión actual. Rev. chil. pediatr. [revista en la Internet].79(1), 13-20.

UNESCO, (2012). Respuestas del sector educación frente al bullying homofóbico. Recuperado de: http://www.unesco.org/new/fileadmin/MULTIMEDIA/FIELD/Havana/pdf/Educacion_bullying.pdf 
